\title{
Horizontal Gene Transfers in Mycoplasmas (Mollicutes)
}

\section{Citti ${ }^{1 *}$, E. Dordet-Frisoni ${ }^{1}$, L.X. Nouvel ${ }^{1}$, $\mathrm{CH} \mathrm{Kuo}^{2}$ and E. Baranowski ${ }^{1}$}

${ }^{1}$ IHAP, Université de Toulouse, INRA, Ecole Nationale Vétérinaire de Toulouse, 23 chemin des Capelles, BP 87614, 31076 Toulouse cedex 3, France

${ }^{2}$ Institute of Plant and Microbial Biology, Academia Sinica, Taiwan

*Correspondence: c.citti@envt.fr

https://dx.doi.org/10.21775/cimb.029.003

\begin{abstract}
The class Mollicutes (trivial name "mycoplasma") is composed of wall-less bacteria with reduced genomes whose evolution was long thought to be only driven by gene losses. Recent evidences of massive horizontal gene transfer (HGT) within and across species provided a new frame to understand the successful adaptation of these minimal bacteria to a broad range of hosts. Mobile genetic elements are being identified in a growing number of mycoplasma species, but integrative and conjugative elements (ICEs) are emerging as pivotal in HGT. While sharing common traits with other bacterial ICEs, such as their chromosomal integration and the use of a type IV secretion system to mediate horizontal dissemination, mycoplasma ICEs (MICEs) revealed unique features: their chromosomal integration is totally random and driven by a DDE recombinase related to the Mutator-like superfamily. Mycoplasma conjugation is not restricted to ICE transmission, but also involves the transfer of large chromosomal fragments that generates progenies with mosaic genomes, nearly every position of chromosome being mobile. Mycoplasmas have thus developed efficient ways to gain access to a considerable reservoir of genetic resources distributed among a vast number of species expanding the concept of minimal cell to the broader context of flowing information.
\end{abstract}

\section{Introduction}

Horizontal gene transfer (HGT) is a major contributor to microbial evolution and adaptation by allowing bacteria to rapidly acquire new traits from external resources. Mechanisms underlying this phenomenon include (i) natural transformation, with the uptake of naked DNA, (ii) transduction with the injection of viral DNA and (iii) conjugation, with conjugative elements as vessels navigating between cells (Frost et al. 2005; Ochman et al. 2000). Network analyses of gene sharing among bacterial genomes suggest that most HGT occurs when donor and recipient are proximate, and hence designate conjugation as the predominant mechanism of HGT (Halary et al. 2010; Kloesges et al. 2011).

Bacterial conjugation is a contact-dependent process actively transferring DNA from a donor, usually carrying a conjugative element, to a recipient cell most often lacking that element. Conjugative systems are encoded by plasmids but chromosomal Integrative and Conjugative Elements (ICE) have been recently recognized as the most abundant conjugative elements in prokaryote (Guglielmini et al. 2011). ICEs are self-transmissible Mobile Genetic Elements (MGE) that encode the machinery for their excision from the host chromosome, their transfer through a conjugative channel and subsequent integration into the recipient where they replicate as a part of the host chromosome (Bellanger et al. 2014). Conjugative plasmids and ICEs most likely have an intricate, common evolution history but differ by their intracellular versus chromosomal residence. Bacterial genomes are also populated with integrative mobilizable elements (IME) that are not self-transmissible per se but can take advantage of other, resident conjugative machineries (Daccord et al. 2010).

Conjugative transfers are not restricted to the movement of plasmids or MGEs across bacterial 
Table 1. Taxonomy of genera included in the Mollicutes class.

\begin{tabular}{|c|c|c|c|c|}
\hline Order & Family & Genus & $\begin{array}{c}\text { Number } \\
\text { of species } \\
\text { (subspecies) }\end{array}$ & Host \\
\hline \multirow[t]{4}{*}{ Mycoplasmatales } & Mycoplasmataceae & Mycoplasma & $119(4)$ & Animal, Human \\
\hline & & "Ca. Mycoplasma" & $13(2)$ & \\
\hline & & Ureaplasma & 7 & Animal, Human \\
\hline & & Eperythrozoon ${ }^{a}$ & 2 & Animal \\
\hline Haloplasmatales & Haloplasmataceae & Haloplasma & 1 & Sea \\
\hline \multirow[t]{3}{*}{ Entomoplasmatales } & Entomoplasmataceae & Entomoplasma & 6 & Insect, Plant \\
\hline & & Mesoplasma & 12 & Insect, Plants \\
\hline & Spiroplasmataceae & Spiroplasma & 36 & Insect, Plants \\
\hline \multirow[t]{2}{*}{ Acholeplasmatales } & Acholeplasmataceae & Acholeplasma & 15 & Animal, Plant \\
\hline & & "Ca. Phytoplasma" b & 38 & Insect, Plant \\
\hline \multirow[t]{2}{*}{ Anaeroplasmatales } & Anaeroplasmataceae & Anaeroplasma & 4 & Bovine, Ovine \\
\hline & & Asteroleplasma & 1 & Bovine, Ovine \\
\hline \multicolumn{5}{|c|}{$\begin{array}{l}\text { (from LPSN http://www.bacterio.net/mollicutes.html, Tully et al. 1993; Johansson and Petterson 2002) } \\
\text { a Most species of the genus Eperythrozoon have been transferred to the genus Mycoplasma except E. coccoides } \\
\text { and E. parvum according to Rules of the Bacteriological Code, Uilenberg et al. IJSB } 2006 \text { proposed that the validly } \\
\text { published names Eperythrozoon and Mycoplasma continue to be used as separate genera possibly in the same } \\
\text { Mycoplasmataceae family } \\
\text { b Genus of uncertain taxonomy composed by in vitro uncultivable species. Phylogenetically near of acholeplasmas, } \\
\text { and provisory classed in the Acholeplasmataceae family. }\end{array}$} \\
\hline
\end{tabular}

cells but can also involve chromosomal DNA regions as initially observed by Lederberg and Tatum in 1946 (Lederberg and Tatum 1946). Using high-frequency recombination $(\mathrm{Hfr})$ E. coli strains, these authors showed the horizontal transfers of genomic DNA, now known as Hfr- or oriT-based transfers. These events are initiated from an integrated origin of transfer (oriT) and are characterized by a gradient, with genes closer to the oriT being more reliably and more frequently transferred (Hochhut et al. 2000), mainly because of physical constraints applying on large molecules during transfer. More recently, chromosomal transfers have been described in Mycobacterium smegmatis (Gray et al. 2013; Derbyshire and Gray 2014) and in Mycoplasma agalactiae (DordetFrisoni et al. 2014) that differ from the classical oriTbased conjugation and thus are not directly associated to MGEs (see also below). In both cases, resulting transconjugants displayed chimeric genomes (see below). In M. smegmatis the situation was extreme, with some transconjugants having acquired up to $25 \%$ of the donor chromosome distributed over 30 loci and the associated mechanism was called Distributive Conjugal Transfer (DCT).

In this review, we described a new family of selftransmissible ICEs discovered in mycoplasmas (MICEs) and the occurrence in these minimal bacteria of chromosomal transfer via an unconventional mechanism that resembles DCT. We further discussed the impacts of these transfers on mycoplasma evolution and on the emergence of new phenotypes with modified virulence traits when pathogenic species are concerned. 


\section{Mollicutes (mycoplasmas) and the theory of reductive evolution}

\section{Mycoplasmas as minimal cells}

Organisms of the Class Mollicutes belong to a large group of wall-less bacteria which taxonomy comprises six families and over 10 genera (see Table 1). In this review, we have mainly concentrated on three genera, which are Mycoplasma, Ureaplasma and Spiroplasma, and have used the trivial term "mycoplasma" to designate any member of the Mollicutes.

From a phylogenetic point of view, mycoplasmas have derived from Gram positive bacteria with low GC content by a process called reductive evolution, which was marked by successive and drastic genetic losses. As a result, current mycoplasmas are characterized by a small genome (ca. 0.5 to 2.0 $\mathrm{Mbp}$ ), the total lack of a cell-wall and reduced metabolic pathways. With the exception of phytoplasmas (Table 1) and a few Mycoplasma species, most mycoplasmas are able to selfreplicate under laboratory conditions in axenic, complex media. Of note, bacteria with smaller genomes (genome size of ca. 0.1 to $0.7 \mathrm{Mbp}$ ) have been identified but these are obligate endosymbionts that do not seem to be able to survive outside of their host cells (McCutcheon and Moran 2011; Moran and Bennett 2014). Because of their small size, mycoplasma genomes were among the first to be fully sequenced, starting in 1995 with the human pathogen Mycoplasma genitalium that displays the smallest gene-set known to support autonomous life. For the very same reason, these minimal bacteria have been used as a blue print for generating the first bacterial cell controlled by a chemically synthesized genome, $M$. mycoides JCVIsyn1.0 (Gibson et al. 2010).

\section{Mycoplasmas as minimal pathogens}

Despite a limited genetic content, a large number of mycoplasmas have emerged and are emerging as important pathogens of man, animals, insects and plants. In human and veterinary medicine, several Mycoplasma and Ureaplasma species are recognized pathogens, often associated with slowly progressive or chronic diseases (Razin and Hayflick 2010; Citti and Blanchard 2013). Most of these have a predilection for the mucosal of the respiratory and the genital tract, causing respiratory diseases and genital disorders, or for the joint, causing arthritis. These pathogenic species have developed common strategies to colonize and circumvent their immunocompetent hosts yet there is no correlation between their phylogenetic position (Figure 1) and a particular degree of virulence or host. For instance, the Hominis group includes species that are pathogenic either for ruminants such as $M$. agalactiae responsible of mastitis, pneumonia and arthritis in sheep and goat, or for swine such as $M$. hyopneumoniae responsible of enzootic pneumonia in pig, or for poultry with $M$. synoviae, that is associated to synovitis and/or airsacculitis. A similar observation can be made for the Pneumoniae or for the Spiroplasma groups. This latter group is remarkable because it includes several major ruminant pathogens, such as $M$. mycoides subsp. mycoides or $M$. capricolum capripneumoniae, along with Spiroplasma species (Gasparich et al. 2004) that are pathogenic for plants and/or insects. For instance, S. citri and S. kunkelli of the Citri clade are two insect-transmitted phytopathogens, responsible of the citrus stubborn and the corn stunt diseases, respectively. The Apis clade includes several species pathogenic for insects such as $S$. apis for honeybees or $S$. culicicola for mosquitoes. Several species across multiple clades are pathogenic for aquatic crustaceans such as $S$. penaei (Nunan et al. 2005) of the Citri clade for saltwater shrimps and $S$. eriocheiris (Wang et al. 2011) of the Mirum clade for freshwater crabs. For more intimate associations with their hosts, some Spiroplasma lineages have become vertically-transmitted reproductive parasites with male-killing effects. This is the case of the Drosophila-associated S. poulsonii (Citri clade) and at least one unnamed lineage in the Ixodetis clade infecting ladybird beetles (Tinsley and Majerus 2006). Interestingly, some Spiroplasma spp. have evolved as defensive mutualists providing their Drosophila hosts with a protection against nematodes (Jaenike et al. 2010) or parasitic wasps (Xie et al. 2010, 2011). Finally, only one Spiroplasma species is known as a pathogen of vertebrates (Tully 1982) namely S. mirum, the suckling mouse cataract agent (Tully et al. 1976).

Because of their limited coding capacity, most mycoplasmas have adopted a parasitic life-style and live in intimate contact with their hosts in which they often act as facultative intracellular symbionts. Recently, Mollicutes-related endobacteria (MRE) have been described as representing a sister clade of the Mycoplasmatales and Entomoplasmatales, within the Mollicutes (Naumann et al. 2010; Naito et al. 2015; Torres-Cortés et al. 2015). These organisms are obligate symbionts of arbuscular mycorrhizal fungi (AMF), which have themselves formed intimate, mutualistic symbioses with the vast majority of land plants, providing them with mineral 


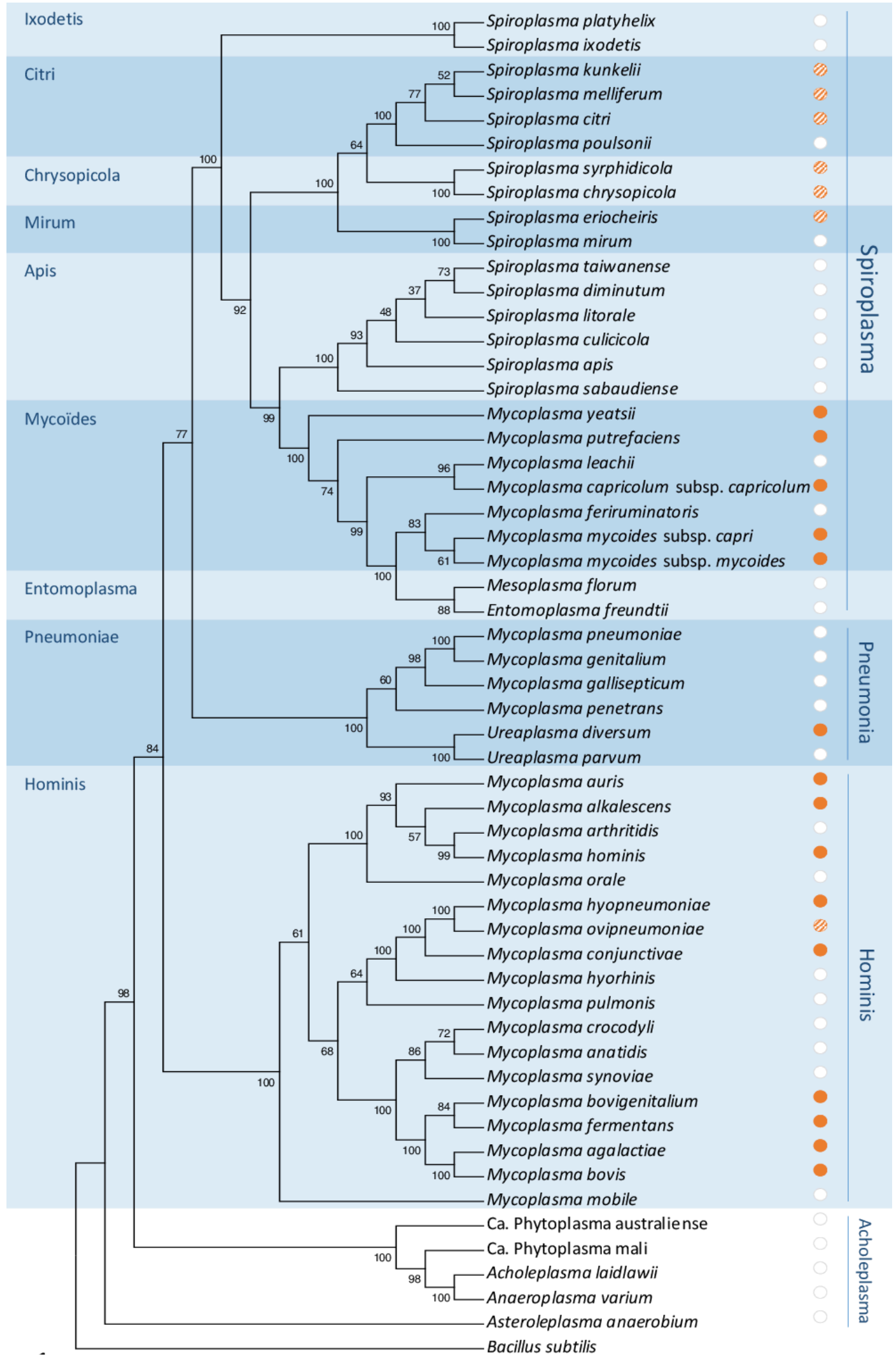


Figure 1. Evolutionary relationships of selected mycoplasmas (Mollicutes) and MICE distribution

The phylogenetic tree was inferred from 16S rDNA sequences using the Neighbour-Joining method (Saitou and Nei 1987). Phylogenetic clades commonly used in this manuscript are indicated on the left and the four main phylogenetic groups are indicated on the right side. The bootstrap consensus tree was inferred from 500 replicates and is taken to represent the evolutionary history of the taxa analysed (Felsenstein 1992). Branches corresponding to partitions reproduced in less than $50 \%$ bootstrap replicates are collapsed. The percentage of replicate trees in which the associated taxa clustered together in the bootstrap test (500 replicates) are shown next to the branches (Felsenstein 1992). The evolutionary distances were computed using the Maximum Composite Likelihood method (Tamura et al. 2004) and are in the units of the number of base substitutions per site. The analysis involved 55 nucleotide sequences. All positions containing gaps and missing data were eliminated. There were a total of 1159 positions in the final dataset. Evolutionary analyses were conducted in MEGA7 (Kumar et al. 2016). The distribution of MICE or MICE factors is indicated on the right side: full orange dots indicate species for which ICE were previously described in at least one strain; hatched orange dots designate species for which both CDS5 and CDS17 were concomitantly detected; white dots correspond to species for which no MICE has been reported or for which the concomitant occurrence of CDS5 and CDS17 was not detected. CDS5 and CDS17 were searched by blast analysis using standard parameters and CDS5 and CDS17 amino acid sequences of M. agalactiae (MAGA7030 and MAGA6970 respectively) and M. mycoides subsp. capri GM12 (MMCAP2_0555 and MMCAP2_0567, respectively), all available at the $\mathrm{NCBI}$ public database (https://www.ncbi.nlm.nih.gov/).

nutrients and water, receiving in return carbohydrates from photosynthesis. These MRE are found in most AMF species, indicating that their associations have an ancient origin. However, the biological functions of MRE are unknown.

\section{Horizontal gene transfers in mycoplasmas}

For decades, genome streamlining was thought to be the only force driving mycoplasma evolution. Indeed, HGT was thought to be marginal in these organisms because of their limited content in efficient recombination systems (Rocha et al. 2005) and in prophage or conjugative elements as means for DNA uptake (Sirand-Pugnet et al. 2007a, b). Interest in mycoplasmas HGT was revived by the advances in sequencing: comparative genomics revealed that large chromosomal DNA exchanges have taken place between phylogenetically remote mycoplasma species known to colonize the same habitat (Sirand-Pugnet et al. 2007b; Pereyre et al. 2009). The concomitant detection of putative ICEs (Calcutt et al. 2002; Marenda et al. 2006) in these species further raised the prospect that these simple bacteria might be able to conjugate. Addressing this issue in mycoplasmas that are fastidious to grow and genetically difficult to manipulate was not trivial and only two early works were supportive of this hypothesis. The first one was based on phenotypic observations of resistance transfer in $S$. citri mutants (Barroso and Labarère 1988) and the second described a DNase-resistant transfer of transposon in M. pulmonis (Teachman et al. 2002). The confirmation that modern mycoplasmas have retained a form of sexual competence and are able to exchange DNA via conjugation further came from the study of ICEs in ruminant mycoplasma species, using the ruminant pathogen $M$. agalactiae as model.

\section{Mycoplama Integrative Conjugative Elements (MICEs): a new family of self-transmissible element with conjugative properties}

Mycoplasma ICEs (MICEs) belong to the large family of bacterial ICEs also designated as conjugative transposons. Over the last decades, ICEs have been increasingly appreciated for their role in HGT (Burrus and Waldor 2004; Delavat et al. 2017) and their importance in the evolution of microorganisms is underlined by their wide distribution across diverse bacteria (Wozniak and Waldor 2010; Guglielmini et al. 2011). These genetic elements are defined by two features: their integration in the host-chromosome and their capacity to encode a type IV secretion system that mediates their transfer from donor to recipient cells via conjugation (Johnson and Grossman 2015). One of the best-known ICEs is Tn916, which is a promiscuous transposon of Enterococcus fecalis that confers resistance to antimicrobials (Roberts and Mullany 2011) and has been introduced by transformation in a number of mycoplasmas (Voelker and Dybvig 1996; Clewell 2008). Whether Tn916 can self-disseminate horizontally in natural conditions to and among mycoplasmas is not known but several genital mycoplasmas resistant to tetracycline possess a tet(M) determinant displaying high identities $(\sim 97 \%)$ to that carried by the Streptococcus pneumoniae Tn916 (Mardassi et al. 2012; Calcutt and Foecking 2015).

\section{MICEs gene content and organisation}

MICEs were first described in two mycoplasma species belonging to the Hominis phylogenetic group (Figure 1): $M$. fermentans, an opportunistic human pathogen and $M$. agalactiae, an important pathogen of small ruminants. M. fermentans ICE, 
MICE backbone

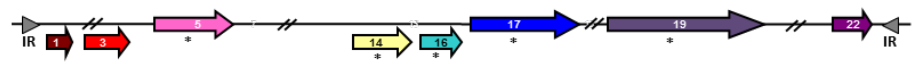

Classical MICE

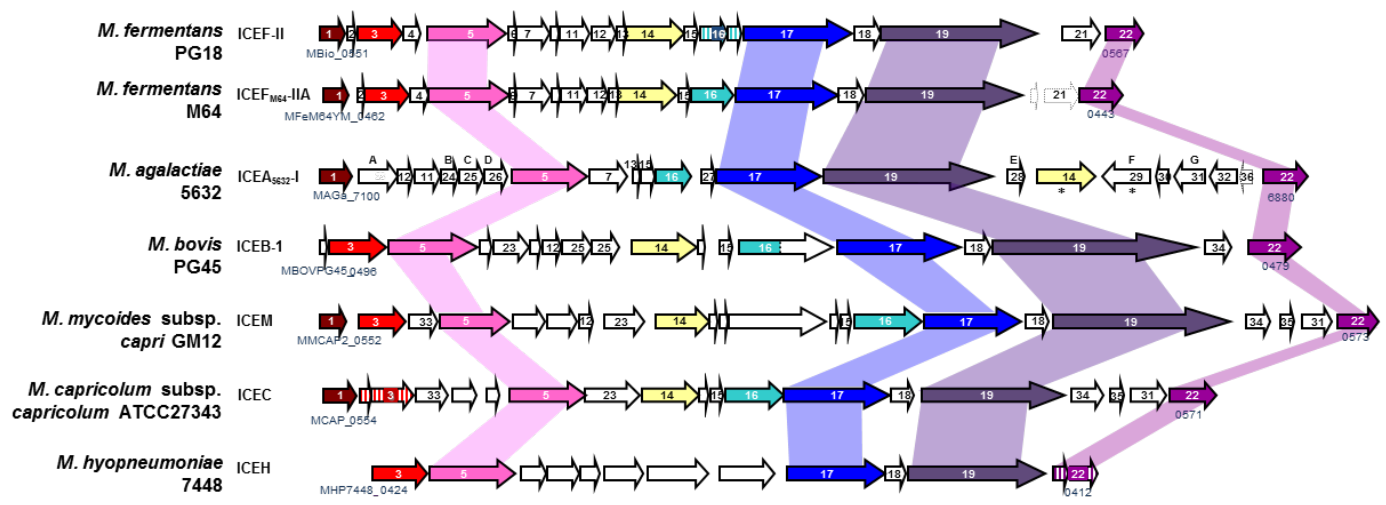

Exemples of degenerated vestigial MICEs

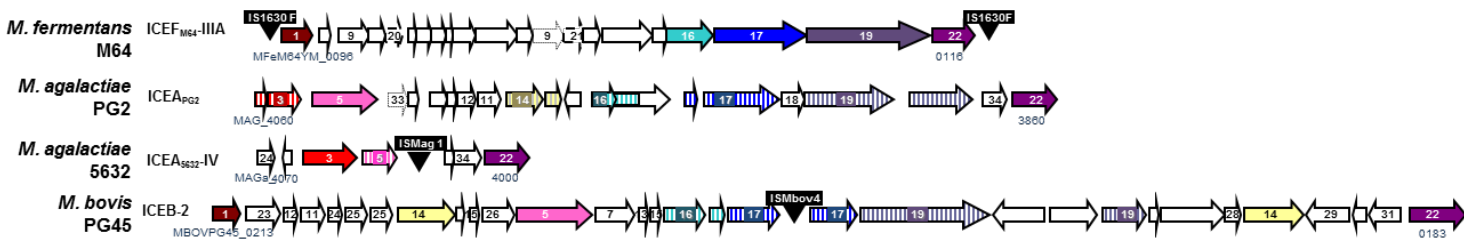

Figure 2. Mycoplasma Integrative and Conjugative Element (MICE) backbone and structural organization of major MICE representatives. In MICE backbone, genes highly conserved across MICEs are positioned on the MICE (solid line) and are represented by colored filled arrows; conserved CDS that may be absent or truncated in particular MICEs are represented below the line. Inverted repeats (IR) are indicated by grey triangles. In classical MICE and degenerated vestigial MICE, highly conserved CDS are colored as in MICE backbone. Pseudogenes are represented by arrows filled with hatched color accordingly to MICE backbone. Genbank unique locus tag numbers of the first and the last MICE genes are indicated in blue below the MICE. Genes containing one or several transmembrane domains are indicated with an asterisk. Insertion sequences (IS) are represented by black boxes.

ICEF, (Calcutt et al. 2002) and M. agalactiae ICE, ICEA, (Marenda et al. 2006) are very similar, large modular elements of approximately 20-30 kbp (Figure 2), occurring as multiple copies in their hostgenomes. Both MICEs are composed of about 20 structural genes with only three (namely cds5, cds12 and cds17) having predicted functional domains common to proteins with DNA mobilityrelated functions in other bacteria (Alvarez-Martinez and Christie 2009). These are (i) a TraG/VirD4 homolog (CDS5, pfam accession number PF02534) that usually couples the relaxome to the conjugal mating pore (Balzer et al. 1994; Llosa et al. 2002), (ii) a single stranded DNA binding (SSB) protein (CDS12, pfam accession number PF00436) and (iii) a TraE/VirB4 homolog (pfam accession number PF05309) usually involved in DNA transport (Komano et al. 2000; Rabel et al. 2003).

With the advances in genome sequencing, several other MICEs have since been described which overall organization is relatively similar (Calcutt et al. 2002; Pinto et al. 2007; Nouvel et al. 2010; Wise et al. 2011; Thiaucourt et al. 2011; Shu et al. 2012) as illustrated in Figure 2 where classical MICEs are depicted. These are composed of approximately 20 genes similarly orientated and share a common minimal backbone consisting of cds5, cds17, cds19 and cds22 (Tardy et al. 2015), a DDE recombinase involved in MICE excision and integration (DordetFrisoni et al. 2013b; Guérillot et al. 2014). Additionally, several other putative structural genes may be included because they occur at a very similar location across a majority of documented MICEs. These are cds 1 and cds3 usually located at the MICE 5'end and cds 14 and cds 16 located upstream of $c d s 17$. Finally, two inverted repeats (IR) flanked the integrated minimal backbone which ones are juxtaposed in the free circular form (see below) (Dordet-Frisoni et al. 2013b). 


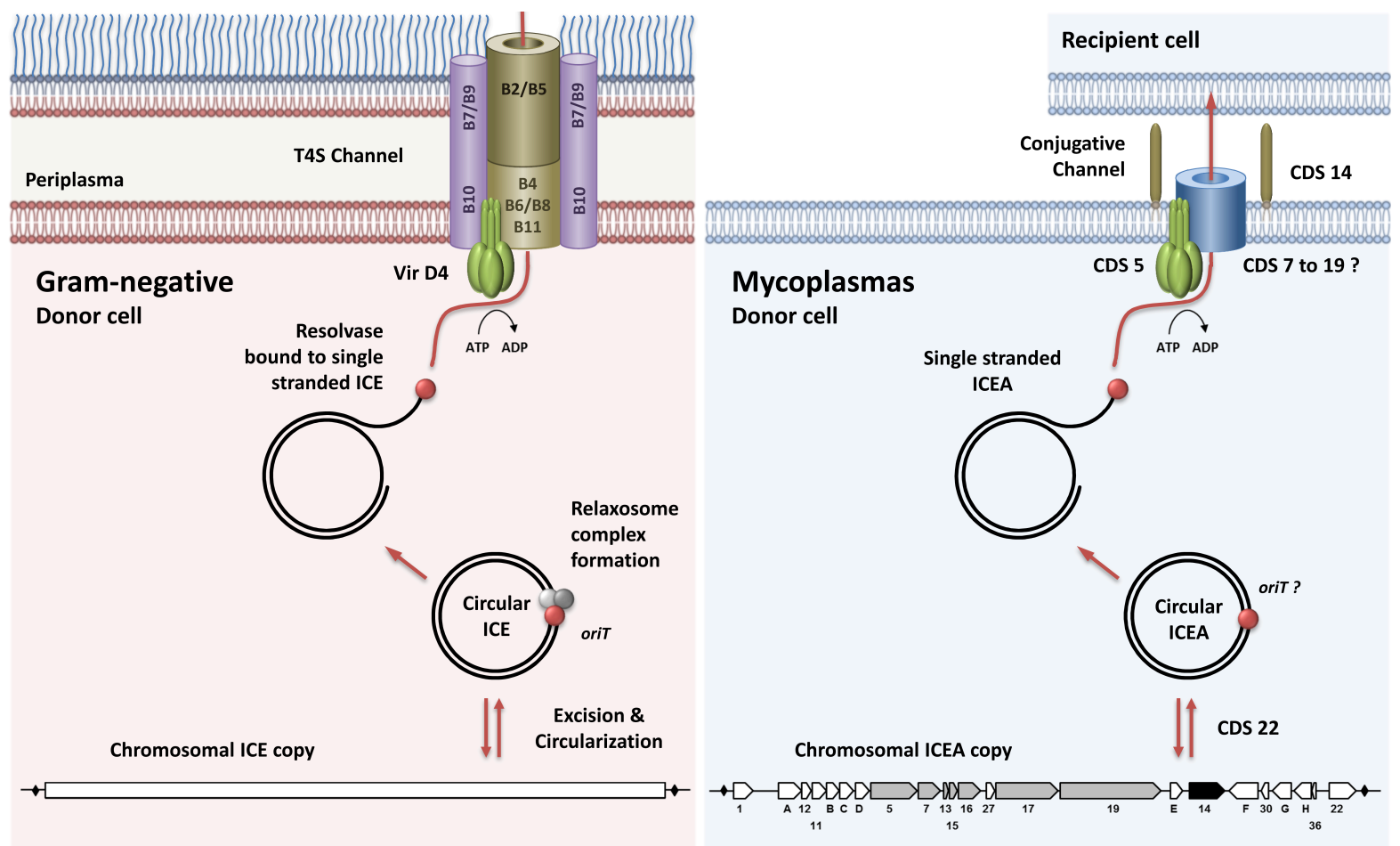

Figure 3. ICE transfer in mycoplasmas. Overview of conjugative transfer in Gram-negative bacteria (Left panel) and proposed model for ICEA transfer in M. agalactiae (Right panel). Bacterial conjugation requires a close contact between the donor and recipient cells. This initial contact often involves complex structures, such as conjugative pili, in Gram-negative bacteria, but only a single surface lipoprotein (CDS14 product) has been so far identified as contributing to this intimate interaction in M. agalactiae. Upon induction, by specific cellular conditions or stochastic events, ICEs excise from the chromosome and forms a circular dsDNA molecule. In M. agalactiae, these two events are mediated by a DDE recombinase (CDS22 product). Following circularization, ICE-encoded proteins are actively expressed and assemble into the relaxosome complex at the origin of transfer (oriT), which process the circular ICE to generate a linear ssDNA-protein complex. ICEA circularization also induces the expression of the conjugative module (CDSs in gray) whose products assemble into the mating pore (conjugative channel), a simplified form of T4SS found in Gram-negative bacteria. Finally, the transfer of the single stranded ICE through the conjugative channel is facilitated by the TraG/VirD4 energetic component (CDS5 product) found at the inner side of the membrane. Once in the recipient strain, the ICE re-circularizes, becomes double stranded and integrates into the host chromosome. This schematic was adapted from Alvarez-Martinez and Christie (2009) and represents our current working model of conjugative transfer in mycoplasmas.

In other bacteria, the global ICE structure consists of three modules that enable its maintenance, dissemination, and regulation. Little is known regarding the functionality of MICE components but the clustering of seven genes encoding putative proteins with transmembrane domains (in ICEA CDS5 to CDS19) most likely represents the module associated with the conjugative channel (see also below Figure 3).

Blast searches also revealed the presence of shorter and putatively degraded MICEs in mycoplasma genomes, with some being limited to two intact genes (see Figure 2, ICEA $5632-\mathrm{IV}$ ). As shown in Figure 2, they often carry pseudogenes that most likely reflect past MICE attacks followed by MICE erosion and are thus commonly refer as MICE vestige (VMICE). For instance, diverse VMICEs have been detected in $M$. agalactiae with strain 5632 having two short vMICEs $(<5 \mathrm{kbp})$ in addition to the three complete ICEA copies and PG2 having a longer vestige (ca. $22 \mathrm{kbp}$ ), vICE-PG2, composed of numerous pseudogenes. Several vMICEs, such as ICEB-2 of $M$. bovis, also harbor IS elements, with some being inserted in MICE backbone genes. The accumulation of disruptive mutations and IS supports the assumption of VMICE being non-functional and slowly decaying. Indeed, experimental studies have shown that VICE-PG2 is not active and is not horizontally transferred under laboratory conditions (Dordet-Frisoni et al. 2013b). Nevertheless, the occurrence of vMICEs in a large 
number of species, the lack of functional studies to identify MICE single gene function and the poor sequence similarities with other bacterial systems raised two issues. The first relates to the criteria used for defining MICE during genome analyses. For instance, ICEH of $M$. hyopneumoniae has been included in Figure 2 because it has been described as such but with only 12 genes ICEH stands apart (Pinto et al. 2007). ICEH displays a minimal MICE backbone in which CDS22 appears to be disrupted and was shown to excise from its host chromosome. Since CDS22 encodes the DDE recombinase responsible for MICE excision this data is puzzling. The second question thus relates to vMICEs possibly still maintaining functions that may be activated or complemented under certain circumstances. In this review, MICEs were designated as such when displaying the minimal backbone (as defined above) within a locus of ca 30 kbps (see Figure 2) and/or there were some experimental evidences for their functional activity.

MICEs were mainly identified in the Hominis and the Spiroplasma phylogentic groups (Figure 1). A current blast search retrieved the presence of CDS5 and CDS17 homologs in Ureaplasma of the Pneumoniae group such as in the $U$. diversum genome of strain ATCC 49782 (Marques et al. 2015, 2016, see also below) but not in Phytoplasma which include non-cultivable species. Within the Spiroplasma phylogenetic group, MICEs occur within the Mycoides clade (Figure 1) and display a global nucleotide identity ranging between 68 and $89 \%$. These include ICEC and ICEM respectively found in $M$. capricolum subsp. capricolum and in $M$. mycoides subsp capri, (Sirand-Pugnet et al. 2007b; Thiaucourt et al. 2011). Putative homologs of traG and/or traE (also designated traE) have been found in several Spiroplasma genomes (Saillard et al. 2008; Ku et al. 2013; Lo et al. 2013b; Paredes et al. 2015; Lo et al. 2015) but the occurrence of functional MICEs has yet to be formally addressed in this genus. In S. citri, the situation is complex with traG and traE homologs being carried both by plasmids (Saillard et al. 2008) and by the chromosome (Carle et al. 2010).

Interestingly, the Mycoides clade is composed of mycoplasma species infecting ruminants, with some sharing the same in-host niches with $M$. agalactiae or $M$. bovis. Based on their gene content, organization and sequences, MICEs found in ruminant species can be divided in two types that match with their phylogenetic position: the hominis and the spiroplasma types, with ICEA of strain 5632 and ICEM of strain GM12 as respective prototypes. This subdivision is particularly appropriate for the CDS22 transposase that is highly conserved within each MICE type but not in between types.

Small plasmids (ca. 2kbps) were found in mycoplasma of the Mycoides clade (Breton et al. 2012) together with MICEs raising the question of their interaction. In M. mycoides subsp. capri, an identical short non-coding region (ca. $150 \mathrm{nt}$ ) is present on both MICEs and plasmids (Thiaucourt et al. 2011), offering a means for recombination (Wozniak and Waldor 2010). Such co-integrates have yet to be isolated experimentally but they could provide small plasmids with MICE conjugative properties for their horizontal dissemination. In contrast, no plasmid was found in ruminant mycoplasma species of the Hominis phylogenetic group.

MICEs abundance in the two phylogenetic groups, their conjugative properties point towards these mobile elements as the main contributors, contrary to plasmids, of the massive HGT previously detected by computational analyses between $M$. agalactiae (Hominis group) and members of the Mycoides cluster (Spiroplasma group) (SirandPugnet et al. 2007b).

\section{MICE horizontal transmission}

MICE propagation is secured by passive, vertical transmission during chromosomal replication and cell division. Functional studies performed with $M$. agalactiae have shown that ICEA is also able to self-disseminate horizontally via conjugation, a cellto-cell process occurring at a low frequency (ca 10-7 transconjugants/donor strain) (Dordet-Frisoni et al. 2013b). Circumstances triggering this rare event are not known but we suspect that, as shown for other bacteria, chromosomal ICE genes are most often silent (see also below).

For cell-to-cell transfer, MICEs must first free themselves from the host chromosome. In $M$. agalactiae, this step involves short inverted repeats (IR, GGAA/TTCCC) located at the left and right end of the ICE which are then juxtaposed in the circular, extrachromosomal form after ICE excision. Free, circulate intermediates as well as empty chromosomal sites have been detected by PCR in several mycoplasmas species bearing ICEs (Calcutt et al. 2002; Vasconcelos et al. 2005; Marenda et al. 2006; Pinto et al. 2007; Tardy et al. 2015). MICE excision and subsequent integration in the new recipient chromosome is driven by a DDE 
recombinase, CDS22, which gene is located at one end of the element (Dordet-Frisoni et al. 2013b; Guérillot et al. 2014). DDE recombinases are typically associated with IS, transposon or phage and do not usually target specific site for integration (Brochet et al. 2009). Indeed, ICEA integration into the recipient chromosome is totally random, occurring at different loci, mostly in putative coding regions. This was demonstrated by direct sequencing of host-chromosomal regions that flanked the integrated ICE in a series of transconjugants derived from a single pair. As well, random chromosomal insertions of a mini-ICE construct that carried cds22 and a selection maker flanked by ICEA inverted repeats were obtained upon transformation of a $M$. agalactiae ICE-negative strain (Dordet-Frisoni et al. 2013b). Integration of MICE circular forms results in the duplication of $8 \mathrm{nt}$ of the host chromosome that end up on each side of the element as direct repeats, an event leaving no trace after ICE excision (Calcutt et al. 2002; Marenda et al. 2006; Dordet-Frisoni et al. 2013b). In $M$. agalactiae, close examination of the ICEA integration sites revealed no polymorphism indicative of the presence of the donor chromosome other than that encoded by the ICE suggesting a clean cut and paste mechanism. Indeed, extrachromosomal circular forms of ICEA as well as chromosomal empty sites were detected in all ICEA transconjugants tested so far. Transient replication of ICE-circulate intermediates has been described for some bacteria hosting mobile elements associated with DDE transposases (Brochet et al. 2009) but has yet not been detected in mycoplasmas. The two streptococcal ICEs, TnGBS1 and TnGBS2, also encode DDE recombinase and have no specific target site but preferentially insert upstream of promoter sequences (Brochet et al. 2009). Overall, this contrast with the majority of bacterial ICEs described so far that use tyrosine recombinase, which one mediates site-specific recombination and often targets a specific attachment site (att) in the bacterial chromosome (Johnson and Grossman 2015). Altogether, experimental evidences have shown that ICEA is self-transmissible across cells and encode elements involved in its chromosomal excision, integration and in conjugation.

As mentioned earlier, the DDE recombinase, CDS22, is highly conserved within MICE types, the hominis- or the spiroplasma-type, but not in between types. IRs targeted by the recombinase during excision-integration are identical within MICE types but differ in between types, most likely reflecting a tight, specific interaction. Whether the DDE recombinases encoded by $c d s 22$ of the hominis-type are able to act on MICE of the spiroplasma type and vice et versa is not known, but it is interesting to speculate that the two ICE types may differ in their fine excision mechanism. Junctions of free circular MICEs (cMICEs) of the hominis type displayed a conserved sequence while those of the spiroplasma type only shared the same IR.

\section{MICE conjugative machinery}

MICEs transfer to a recipient cell via a conjugative process most likely as a single stranded DNA molecule with the help of SSB proteins. In mating conditions, ICE-negative strains of $M$. agalactiae are unable to undergo HGT unless they have received an ICEA. Thus, ICEA provides the recipient cell with the ability to conjugate, including the ability to exchange chromosomal DNA independently from ICE transfer (see below).

In bacteria, conjugation relies on a type IV secretion system which is expected to be minimal in the wallless mycoplasma cells. In their early work on chromosomal gene transfer in S. citri, Barroso and Labarère (Barroso and Labarère 1988) proposed that HGT might arise through fusion of donor and receptor cell membranes. Later, pili-like appendages were detected on the cell surface of $S$. kunkelii that connected two cells (Ozbek et al. 2003), suggesting that even simplified cells can produce sophisticated structures. Transmission or scanning microscopy of $M$. agalactiae strains, with or without ICE, in mating conditions or not, did not reveal any particular structure such as a pilus (unpublished data). The mycoplasma cells-membranes were however in intimate contact, with some structures resembling the nanotubes described by Dubey and Ben-Yehuda when studying neighboring Bacillus subtilis and Staphylococcus aureus or $B$. subtilis and Escherichia coli cells (Dubey and Ben-Yehuda 2011).

While membrane fusion during conjugation cannot be formally ruled out, several arguments point towards the synthesis of a conjugative channel between donor and recipient cells, as for other bacteria. In ICEA, candidates for the conjugative channel formation include seven gene products with transmembrane domains, namely CDS5, $7,13,15$, 16, 17 and 19 (Figure 3). One additional surface lipoprotein, CDS14 is required for both ICE and chromosomal transfer: inactivation of cds 14 abrogates conjugation, an event which is restored 
upon complementation by the wild-type cds 14 (Dordet-Frisoni et al. 2013b). CDS14 was confirmed experimentally to be expressed at the cell surface and was among the few ICE proteins to be detected by proteomics in standard laboratory conditions before mating (personal data). Interestingly, CDS14 does not have to be expressed by the ICE-donor cell as ICE transfer were documented in between an ICE-positive, donor strain deficient in CDS14 and an ICE-negative recipient strain in which wild-type CDS14 has been introduced. Based on these results, our current hypothesis is that CDS14 contributes to initiating the first conjugative step and is involved in cell-to-cell contact.

\section{MICEs prevalence among mycoplasmas}

The late discovery of MICEs is partly due to their variable occurrence within and among Mollicutes species. Hence, MICEs appear to be completely lacking in some species such as $M$. pneumoniae or M. genitalium of the Pneumoniae phylogenetic group, two human pathogens for which a high number of strains have already been sequenced. In contrast, the Hominis group is rich of mycoplasmas species bearing MICEs such as the human $M$. fermentans which bears several MICEs, the ruminant pathogen $M$. bovis where MICEs or MICE elements are found in all strains with sequenced genome $(n=5)$ or field isolates $(n=42)$ or its closest relative, $M$. agalactiae, with $45 \%$ of the strains ( $n=$ 41) harboring MICE-related sequences (Tardy et al. 2015). Free, circular MICE forms were regularly detected in field isolates of ruminant mycoplasma species that displayed a minimal ICE backbone. Since MICE excision and circularization are the first steps for MICE cell-to-cell transfer (Wozniak and Waldor 2010), isolates currently circulating in ruminants most likely bear MICEs capable of horizontal dissemination.

The Hominis phylogenetic group also contains species so far deprived of MICE such as the avian pathogen $M$. synoviae or the mice pathogen $M$. pulmonis for which only a few sequenced genomes are available in the databases. Finally, no MICE has been detected in the Acholeplasma phylogenetic group.

MICEs also vary in their copy number per hostchromosome, with a single strain having multiple copies of the same ICE, or multiple copies of two different ICEs. This is illustrated by the $M$. fermentans strain M64 which harbours three copies of ICEF-III and three copies of ICE-II (Shu et al. 2012). MICE multiple occurrences complicate their proper identification in mycoplasma genome by providing repeated sequences that hampers correct assembly. Therefore, detailed MICE analyses in draft genomes should be taken with care and further completed by targeted, sequencing strategies. Overall, MICE strain-to-strain variability correlates with their highly dynamic nature and in turn poses the question of their fitness cost on the minimal mycoplasma cell or of their potential benefit, especially for those having multiple copies of similar elements.

\section{MICE evolutionary history and trafficking}

DDE recombinases (CDS22) are key elements of MICE mobility (Dordet-Frisoni et al. 2013b). A cascade of iterated Blast searches using the TnGBS2 transposase of streptococci as primary sequence revealed that MICE DDE recombinases constitute a new family of the Mutator and Mutatorlike superfamily in prokaryotes (Guérillot et al. 2014). Phylogenetic reconstructions further showed that they form a homogeneous, distinct clade, pMULT5 and are related to IS256, a widely spread insertion sequence (Guérillot et al. 2014). IS256related elements most likely circulate in mycoplasmas and have been found in the MICEbearing M. fermentans (ISMf1) (Calcutt et al. 2002). As expected, phylogenetic trees using cds22 and CDS5 sequences from predicted functional MICEs and from MICE vestiges indicate that in addition to vertical inheritance, MICEs are circulating across mycoplasma species and phylum.

The high prevalence of ICEs or vICEs in some mycoplasma species is largely due to their vertical chromosomal inheritance. This is illustrated within the Hominis group in which several species with distinct ecological niche harbor very similar MICEs (or MICE elements such as CDS5 or CDS17) as a result of vertical transmission from a common ancestor. These species include: $M$. fermentans, the human mycoplasma (Calcutt et al. 2002), $M$. hyopneumoniae, a swine pathogen (Minion et al. 2004; Vasconcelos et al. 2005; Liu et al. 2011), and several ruminant species such as $M$. bovis (Wise et al. 2011) and M. agalactiae (Marenda et al. 2006; Nouvel et al. 2010), M. auris (Dordet-Frisoni et al. 2013a), M. alkalescens (Manso-Silván et al. 2013) and M. conjunctivae (Calderon-Copete et al. 2009). The presence of a vICE at the very same position in the genome of $M$. bovis PG45 and $M$. agalactiae PG2 (Wise et al. 2011) indicates that MICEs were acquired in this case, prior to speciation. 
The three ICEA copies of the M. agalactiae 5632 genome were initially detected based on their GC\% (27.5\%), a value lower than the average of the chromosome (29.6 \%). As well, in M. fermentans M64, two slightly different MICEs can be distinguished based on different GC content and gene organisation. So far, detection of MICEs based on their GC\% holds for mycoplasma species of the Hominis group but not for the Mycoides cluster for which MICE and chromosomal GC\% are similar (Marenda 2014). That Mycoides MICEs and chromosome have the same GC content may reflect a long evolutionary association. Traces of past Mycoides-MICE horizontal transfer to the Hominis group can be found in the $M$. agalactiae genome with the VMICE of strain PG2 being phylogenetic more related to ICEM of $M$. mycoides subsp. capri than to ICEA. Altogether, this strongly supports MICE horizontal trafficking among divergent mycoplasmas with the occurrence of multiple HGT events from various donors.

Finally, several reports have also suggested the natural occurrence of the TetM determinant in association with the Tn916 (see above). More recently, sequencing data have revealed the presence of truncated Tn916 within an uncharacterized $25-\mathrm{Kbp}$ transposon which is integrated into the rumA gene as for several closely related conjugative transposons found in streptococci (Calcutt and Foecking 2015). Interestingly, the $36.2 \%$ GC content of this transposon contrasted with the $27.1 \%$ of the core $M$. hominis genome. Whether this large mosaic element has retained competency for horizontal transfer is not known but its excision and circularization in $M$. hominis were supported by experimental evidences (Calcutt and Foecking 2015).

\section{Chromosomal transfer (CT) among mycoplasmas}

In 2007, the first description of large-scale HGT among mycoplasmas sharing the same ecological niche marked a turn and challenged the generally accepted evolutionary scenario in which gene loss is the main driving force of mycoplasma evolution (Sirand-Pugnet et al. 2007b). In this study, comparative genomics together with phylogenetic tree reconstructions revealed that approximately $18 \%$ of $M$. agalactiae genome had undergone HGT with members of the phylogenetically distinct Mycoides cluster. No bias in the GC composition of the transferred genes could be observed that would distinguish them from their ancestors. Although base composition is often considered as a poor indicator of HGT (Koski et al. 2001), one possible explanation for this observation is that the acquired sequences have quickly adjusted to their new genome pattern. In $M$. agalactiae, HGT events often involved chromosomal genes clusters containing 2 to 12 elements with half of them displaying the same organization as in the Mycoides cluster genomes (Sirand-Pugnet et al. 2007b). The majority of these genes encodes for products with unknown function $(40 \%)$ and/or with membrane-associated motifs $(30 \%)$ while $15 \%$ have started to decay and were annotated as pseudogenes. Interestingly, $M$. agalactiae transferred genes were distributed all over the chromosome, a scattering that reflected multiple HGT events and/or extensive shuffling via intrachromosomal recombination events of the alien genes after integration.

With the concomitant discovery of MICEs in both $M$. agalactiae and Mycoides cluster genomes, conjugation quickly emerged as a possible mechanism for DNA transfer in mycoplasmas, with the hypothetical scenario in which MICEs could take along its host flanking regions. The conjugal transfer of marked MICEs was experimentally demonstrated in $M$. agalactiae from ICEA-positive to ICEAnegative cells (see above) but surprisingly no DNA of the donor chromosome was directly associated to MICE horizontal dissemination (Dordet-Frisoni et al. 2013b) (Figure 4). Indeed, observing and documenting the chromosomal transfer (CT) in mycoplasmas required a particular strategy: mating experiments were conducted with mycoplasma cells having the antibiotic selective markers inserted in different parts of the chromosome, outside of ICEA for ICEA-positive cells (Dordet-Frisoni et al. 2014). Large-scale chromosomal DNA exchanges were then documented via conjugation but this event was observed from ICEA-negative to ICEA-positive strains (Figure 4). Again, no ICEA sequence was directly associated with the transferred chromosomal sequences. CTs resulted in replacing large portions of the recipient mycoplasma genome by the donor counterparts via homologous recombination. By combining classical mating experiments with high-throughput next-generation sequencing, the transfer of nearly every position of the mycoplasma chromosome was detected. CTs were also experimentally achieved in between the two related species, $M$. agalactiae and $M$. bovis (Dordet-Frisoni et al. 2014). HGT in between members of the Mycoides clade and the Hominis phylogenetic group remains to be documented in vitro but this is still facing the technical challenge of 


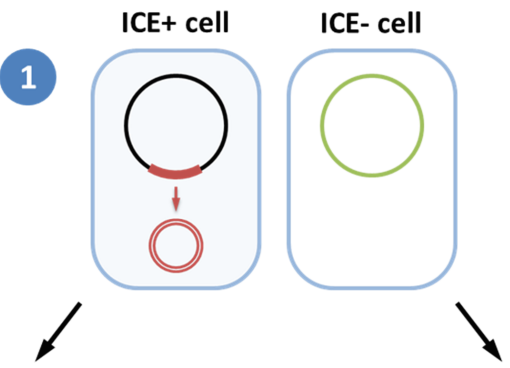

ICE transfer
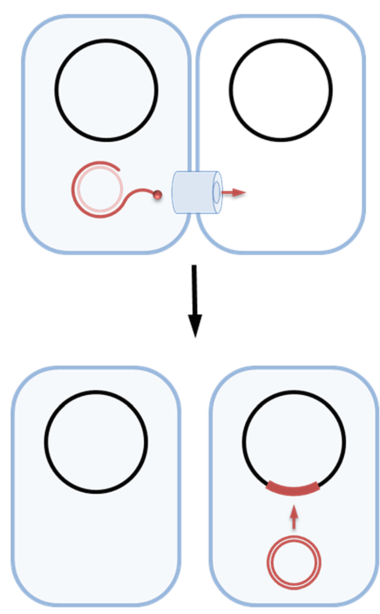

2

Chromosomal exchange
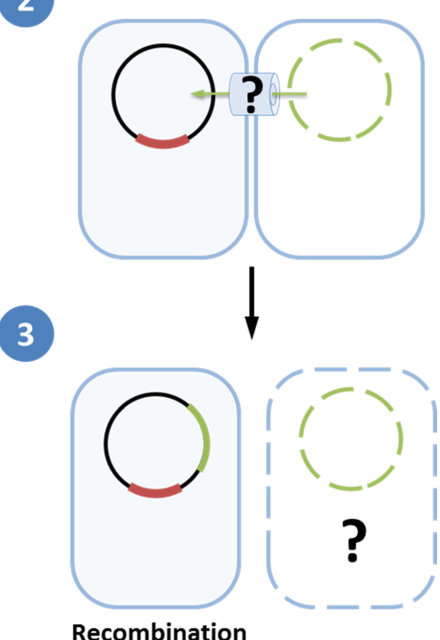

Figure 4. Horizontal gene transfers in mycoplasmas: one ICE, but two mechanisms. Conjugative transfers in mycoplasmas are not restricted to ICE transmission, but can also involve the transfer of large chromosomal DNA fragments. During mating, DNA exchanges occur following two pathways including the horizontal transfer of the ICE (ICE transfer) from ICE-positive (ICE+) to ICE-negative (ICE-) cells and the transfer of chromosomal DNA (Chromosomal exchange) occurring in the opposite direction to the ICE movement. While mechanisms underlying ICE transfer in mycoplasmas share some similarity with the classical pathway (see Figure 3), chromosomal exchanges differ from the classical oriT-based conjugation. While taking advantage of the ICE conjugative machinery, chromosomal exchanges do not rely on the integration of ICE sequences in the transferred chromosomal sequences, but result in DNA swapping of large portions of the recipient mycoplasma genome by the donor counterparts via homologous recombination.

finding laboratory conditions that can support their concomitant growth.

Conjugal transfer of chromosomal DNA is an old theme in bacterial evolution and is usually tightly associated with conjugative elements integrated into the chromosome, such as conjugative plasmids or ICEs, that provides the origin of transfer (oriT). In such system, both the transferred chromosomal segments and the conjugative element initially resided in the same donor cell, a situation contrasting with that described for $M$. agalactiae. As well, mobilization in trans of chromosomal region carrying cryptic oriT (IME) has also been described in several bacteria (Daccord et al. 2010; Bellanger et al. 2014) but again this event is initiated in the same cell. Recently, an IS6-type element carried by a conjugative replicon in Yersinia pseudotuberculosis (Lesic et al. 2012) was shown to drive the horizontal transfer of any large block of plasmid or chromosomal DNA but so far $M$. agalactiae or $M$. bovis are deprived of plasmid (Breton et al. 2012). Bacterial conjugation is classically considered as being unidirectional (Szpirer et al. 1999; Bellanger et al. 2011), but capture of conjugative elements or other cellular DNA by the so called donor has been occasionally reported. Yet, this is a two-steps model requiring first the transfer of the conjugative plasmid or integrative element into the recipient chromosomal DNA that is subsequently retrotransferred back into the donor. This model is appealing because ICEA integration occurs randomly and could theoretically retro-transferred equally any locus using its oriT. Yet it faces several 
inconsistencies such as the observed frequency of chromosomal transfer being much higher than expected in such model. Rather, accumulating evidences point towards a mechanism resembling that described in Mycobacterium smegmatis. In this bacterium, a single recipient chromosome is able to acquire multiple, large unlinked segments of donor DNA (Gray et al. 2013, 2016; Derbyshire and Gray 2014), resulting in mosaic genomes. There is still much to be discovered about this process designated as DCT (distributive chromosomal transfer) but it is associated with the occurrence in the mycobacterium genome of multiple cis-acting sequences termed "bom" regions (basis of mobilization) that initiate transfers. Whether mycoplasmas also possess bom-like regions is not known and the mechanism underlying chromosomal transfer in these minimal bacteria has still to be fully understood. In our hands, two MICE-negative mycoplasma cells are unable to conjugate and no transconjugant is obtained unless the mycoplasma membranes are artificially fused by polyethylene glycol (PEG) (Dordet-Frisoni et al. 2013b, 2014). Thus, our current working model is that CTs in mycoplasma require the presence of MICE in either one of the partners to form the conjugative bridge (Figure 4).

Overall, these finding challenged the classical mechanisms proposed for other bacteria in which CTs are driven by conjugative elements, bringing into the spotlight a new means for rapid mycoplasma innovation. They also radically change our current views concerning the evolution of mycoplasmas, with particularly far-reaching implications given that over 50 species are human or animal pathogens.

\section{Extent of chromosomal horizontal transfer in mycoplasmas and biological impacts}

Thanks to the tremendous advances made in NGS and in comparative genomic analyses, it is becoming now clear that chromosomal HGTs have played a major role in mycoplasma evolution and that this phenomenon is not restricted to ruminant mycoplasmas (see above).

\section{HGT as a means for rapid adaptation}

For instance, HGTs have been described in between the phylogenetic distinct species $M$. hominis and $U$. parvum, two colonizers of the human urogenital tract (Pereyre et al. 2009). These events involved several clusters encoding restriction modification systems, transposases and hypothetical proteins, several of which display motifs associated with surface or membrane exposure and may play a role in the interaction with the host (Pereyre et al. 2009). In silico genome comparative analyses combined to phylogenetic reconstructions also resulted in detecting HGT in between $M$. galisepticum and $M$. synoviae, two poultry pathogens belonging to two distinct phylogenetic groups (Sirand-Pugnet et al. 2007b). Again, these events involved several loci predominantly encoding surface proteins and putative transposases (Noormohammadi et al. 1998; Wang 2001; Vasconcelos et al. 2005; SirandPugnet et al. 2007b).

In absence of a cell-wall, the mycoplasma membrane constitutes the primary interface with the host environment, mediating all types of direct and indirect interactions. It is therefore not surprising that transferred-genes, which were fixed over time, encode proteins with membrane associated motif and most likely are involved in mycoplasma-host interactions. Sharing genetic resources via horizontal gene transfer may provide mycoplasmas with a means for adapting to new niches or to new hosts while avoiding irreversible genome erosion.

\section{HGT between Ureaplasma species questioned serotyping}

Extensive HGTs have also been demonstrated in between human Ureaplasma species and serovars. In a study including over a 1000 clinical isolates of ureaplasmas ( $U$. parvum and $U$. urealyticum), $\mathrm{Li}$ Xiao and co-workers discovered that $40 \%$ of the isolates were genetic mosaics, apparently arising from the recombination of multiple serovars (Xiao et al. 2011). Combined to the extensive comparative genomic analysis performed by Paralanov et al. (2012), their data supported the view that ureaplasmas exist as complex, highly heterogeneous populations rather than as stable serovars, due to HGT naturally occurring in these species. For clinicians, these data explain the extensive crossreactivity observed during serotyping and the lack of consistent association of given serotypes with disease, further implying that pathogenicity of ureaplasmas is unlikely to be associated with individual serovars, as it was speculated for decades (Xiao et al. 2011). Several isolates displayed in their genomes genes often associated to horizontal gene transfer such as traG/D homologs, Tn916 sequences, relaxases/mobilization proteins or homologs to phage integrases (Paralanov et al. 2012), but complete MICE have yet to be identified. 


\section{HGT in Spiroplasma and the emergence of new phenoytpes}

As regards to mycoplasmas infecting plants and insects, comparative and evolutionary genomics studies on Spiroplasma clades that included several species such as $S$. citri, S. chrysopicola, S. mirum and $S$. apis, revealed that the patterns of HGT varied widely (Lo et al. 2016). HGT is highly abundant in the most recent common ancestor of the Mirum clade, with an estimation of $7 \%$ of the $S$. eriocheiris protein-encoding genes coming from HGT (Lo et al. 2015). Interestingly, most of these have been lost in the sister species $S$. atrichopogonis, through accumulation of deletions. Due to the strong mutational bias towards deletions commonly observed in bacterial genomes (Kuo and Ochman 2009a) and the positive selection for the removal of pseudogenes (Kuo and Ochman 2010), losses of newly acquired genes is a common scenario unless these genes contribute to the fitness of their new bacterial host (Kuo and Ochman $2009 \mathrm{~b})$. The long-term survival of horizontally acquired genes in $S$. eriocheiris, a species which has experienced a host switch from terrestrial insects to aquatic crustaceans (Bi et al. 2008; Wang et al. 2011), suggests that some of the novel acquired genes may be involved in the adaptation of a new ecological niche.

Besides the colonization of new niches, HGT could play an important role in the emergence of new strains or new phenotypes including for pathogenic species changes in virulence. This is illustrated in mosquito-associated species of the Apis clade (Lo et al. 2013b; Chang et al. 2014) in which HGT is not as prevalent as for the Mirum or the Citri clades but may have had important consequences. In this clade, documented HGTs involve a virulence factor glpO (Chang et al. 2014) that is inferred as being present in the common ancestor of the Apis clade. This gene was then independently lost in the lineages leading to $S$. diminutum and $S$. sabaudiense, two non-pathogenic species for their mosquito host. In contrast, for two pathogenic species of mosquitos: $S$. culicicola which has maintained the vertically inherited copy and $S$. taiwanense which has acquired the entire gene cluster involved in glycerol metabolism (i.e., glpF$g / p O-g / p K)$ from a donor belonging to the MycoidesEntomoplasmataceae clade. Notably, the horizontally acquired glpF-glpO-glpK in $S$. taiwanense appeared to have been integrated into the transcriptional control of their new host genome (Lo and Kuo 2017).
In spiroplasmas, mechanisms underlying HGT have yet to be elucidated but the presence of tra homologs (see above) together with experimental data (Barroso and Labarère 1988) tend to designate conjugation as one means. Yet a striking feature of the genomes of the Citri clade is the prevalence of sequences related to the plectrovirus genus that can account for ca. $20-30 \%$ of the chromosomes (Carle et al. 2010; Lo et al. 2013a). In addition to facilitating genome rearrangements, invasion of these viral sequences is associated with large fragments (i.e., $>10 \mathrm{~kb}$ ) of novel DNA at the viral insertion sites (Ku et al. 2013), suggesting that HGT in this clade may be mediated via transduction as well.

\section{HGT occurs in mycoplasma sharing a same niche}

A hallmark of mycoplasma DNA exchanges so far described is that donors and recipients colonize the same ecological niche. This is best illustrated by the trans-kingdom transfers recently documented in between the MRE by two independent studies concomitantly describing the occurrence of HGT in between MRE and their fungal host (Naito et al. 2015; Torres-Cortés et al. 2015) although data were obtained with MRE populations associated with different AMF hosts. Briefly, MRE genomes were shown to harbor genes horizontally acquired from AMF that encoded a large repertoire of proteins with eukaryotic domains. For instance, these transkingdom transfers involved genes encoding small ubiquitin-like modifier (SUMO) proteases specific to eukaryotic SUMOylation systems. Although experimental data are needed, fungal SUMO proteases might play an important role for MRE by providing them with a means for manipulating the fungal host cytoplasmic environment in order to increase MRE fitness. Altogether, the large number of acquired genes with eukaryotic domains suggests the MRE high degree of adaptation to their fungal host (Kuo 2015; Naito et al. 2015; Torres-Cortés et al. 2015).

While sharing the same ecological niche is an obvious facilitator of HGT, other environmental or genetic factors such ICEs are required. For instance, $M$. genitalium also colonizes the human urogenital but there is no evidence of HGT with the $M$. hominis or $U$. parvum co-resident species. Like M. genitalium, M. pneumoniae appears to be deprived of MICEs and a recent comparative genomic analysis of strains isolated between 1940 to 2009 indicate that its genome is extraordinarily stable over time and geographic distance across the 
globe with a striking lack of evidence of horizontal gene transfer (Xiao et al. 2015).

Finally, detecting Mycoplasmas HGT by in silico comparative genomic and phylogenetic reconstruction is often difficult in between closely phylogenetically related organisms or within the same species. Thus, our current view is most likely underestimating the role of these events.

\section{Conclusions and future challenges}

Despite having a minimal genome, several mycoplasmas have retained a form of sexual competence and are able to exchange significant amount of chromosomal material in addition to MGEs. This finding is radically changing the old paradigm of mycoplasma evolution being only driven by gene losses. Naito and Pawlowska (Naito and Pawlowska 2016) have recently proposed that this sexual competence is crucial for obligate intracellular mycoplasmas such as MREs to defy the effects of Muller's ratchet, a process in which accumulation and fixation of deleterious mutations drives to extinction small, asexual populations. Pathogenic intracellular or extracellular mycoplasma populations have to face various host responses and hostile environments during dissemination and colonization, going through continual bottlenecks. Their limited genetic material and lack in DNA repair components make them particularly vulnerable to the deleterious effect of Muller's ratchet. HGT and the capacity to horizontally exchange any part of their genome may contribute to the maintenance of genomic information in mycoplasma subpopulations, providing them with a means to rescue injured genomes, restoring deleted or inactivated genes.

Except for genes that are lethal for the recipient mycoplasma cell, virtually any essential or accessory gene can be transferred. Thus, mycoplasma populations can be seen as a dynamic gene pool compensating genome erosion as well as clonality, allowing horizontal dissemination of genetic traits that may in turn result in the emergence of new strains, with new properties. While this may be beneficial to the survival of the minimal mycoplasma cells, such capacity may slow down the control of mycoplasma infectious diseases via the spreading genetic material associated to antibiotic resistance or virulence.

Understanding the mechanisms underlying and influencing mycoplasma HGT is an important issue but has just started to be explored. Since only conjugative transfers have been formally demonstrated so far, cell-to-cell contact constitutes the primary constraint for mycoplasma HGT to take place. Another is the occurrence of MICE in at least one partner to provide the conjugative pore (Figure 4). Indirect evidences also point towards additional HGT mechanisms such as transduction for spiroplasmas but the role of viruses in this phenomenon has yet to be addressed.

Finally, in mycoplasmas, viable conjugal transfers are detected at very low frequencies $\left(10^{-7}\right)$ under laboratory conditions most likely because of MICE expression being shut down. MICE excision and subsequent horizontal transfers might be modulated by external factors such as physical stresses (i. e. temperature) (Tardy et al. 2015), or by host- or MICEs-genetic factors. Our current hypothesis is that CTs are intimately linked to the MICE-encoded conjugal pore, thus deciphering the environmental and mycoplasma factors that can trigger or repress MICE expression is currently emerging as a primary question.

\section{Acknowledgements}

Due to length constraints, many references could not be cited. Our work on the role of MGE and on chromosomal transfer was supported by the French National Research Agency, ANR, (MycXGene, ANR09MIE016 coordinated by CC), by the National Institute for research in Agriculture (INRA), and by the national veterinary school of Toulouse (ENVT). Funding to $\mathrm{CHK}$ was provided by Academia Sinica (Taipei, Taiwan).

\section{References}

Alvarez-Martinez CE, Christie PJ (2009) Biological diversity of prokaryotic type IV secretion systems. Microbiol Mol Biol Rev MMBR 73:775-808. doi: 10.1128/MMBR.00023-09

Balzer D, Pansegrau W, Lanka E (1994) Essential motifs of relaxase (Tral) and TraG proteins involved in conjugative transfer of plasmid RP4. J Bacteriol 176:4285-4295

Barroso G, Labarère J (1988) Chromosomal gene transfer in Spiroplasma citri. Science 241:959-961

Bellanger X, Morel C, Gonot F, et al (2011) Site-specific accretion of an integrative conjugative element together with a related genomic island leads to cis mobilization and gene capture. Mol Microbiol 81:912-925. doi: 10.1111/j.1365-2958.2011.07737.x

Bellanger X, Payot S, Leblond-Bourget N, Guédon G (2014) Conjugative and mobilizable genomic islands in bacteria: evolution and diversity. FEMS Microbiol Rev 38:720-760. doi: 10.1111/1574-6976.12058 
Bi K, Huang H, Gu W, et al (2008) Phylogenetic analysis of Spiroplasmas from three freshwater crustaceans (Eriocheir sinensis, Procambarus clarkia and Penaeus vannamei) in China. J Invertebr Pathol 99:57-65. doi: 10.1016/j.jip. 2008.06.008

Breton M, Tardy F, Dordet-Frisoni E, et al (2012) Distribution and diversity of mycoplasma plasmids: lessons from cryptic genetic elements. BMC Microbiol 12:257. doi: 10.1186/1471-2180-12-257

Brochet M, Da Cunha V, Couvé E, et al (2009) Atypical association of DDE transposition with conjugation specifies a new family of mobile elements. Mol Microbiol 71:948-959. doi: 10.1111/ j.1365-2958.2008.06579.x

Burrus V, Waldor MK (2004) Shaping bacterial genomes with integrative and conjugative elements. Res Microbiol 155:376-386. doi: 10.1016/j.resmic.2004.01.012

Calcutt MJ, Foecking MF (2015) An excisioncompetent and exogenous mosaic transposon harbors the tetM gene in multiple Mycoplasma hominis lineages. Antimicrob Agents Chemother 59:6665-6666. doi: 10.1128/AAC.01382-15

Calcutt MJ, Lewis MS, Wise KS (2002) Molecular genetic analysis of ICEF, an integrative conjugal element that is present as a repetitive sequence in the chromosome of Mycoplasma fermentans PG18. J Bacteriol 184:6929-6941

Calderon-Copete SP, Wigger G, Wunderlin C, et al (2009) The Mycoplasma conjunctivae genome sequencing, annotation and analysis. BMC Bioinformatics 10 Suppl 6:S7. doi: 10.1186/1471-2105-10-S6-S7

Carle P, Saillard C, Carrère N, et al (2010) Partial chromosome sequence of Spiroplasma citri reveals extensive viral invasion and important gene decay. Appl Environ Microbiol 76:3420-3426. doi: 10.1128/AEM.02954-09

Chang T-H, Lo W-S, Ku C, et al (2014) Molecular evolution of the substrate utilization strategies and putative virulence factors in mosquito-associated Spiroplasma species. Genome Biol Evol 6:500-509. doi: 10.1093/gbe/evu033

Citti C, Blanchard A (2013) Mycoplasmas and their host: emerging and re-emerging minimal pathogens. Trends Microbiol 21:196-203. doi: 10.1016/j.tim.2013.01.003

Clewell D (2008) Conjugative transposon Tn916 and related elements. In: SciTopics. http://www.scitopics.com/ Conjugative_Transposon_Tn916_and_Related_Element s.html. Accessed 3 Feb 2012

Daccord A, Ceccarelli D, Burrus V (2010) Integrating conjugative elements of the SXT/ R391 family trigger the excision and drive the mobilization of a new class of Vibrio genomic islands. Mol Microbiol 78:576-588. doi: 10.1111/j. 1365-2958.2010.07364.x

Delavat F, Miyazaki R, Carraro N, et al (2017) The hidden life of integrative and conjugative elements. FEMS Microbiol Rev 41:512-537. doi: 10.1093/femsre/fux008

Derbyshire KM, Gray TA (2014) Distributive conjugal transfer: New insights into horizontal gene transfer and genetic exchange in Mycobacteria. Microbiol Spectr 2:. doi: 10.1128/microbiolspec.MGM2-0022-2013

Dordet-Frisoni E, Baranowski E, Barré A, et al (2013a) Draft genome sequences of Mycoplasma auris and Mycoplasma yeatsii, two species of the ear canal of caprinae. Genome Announc 1:. doi: 10.1128/genomeA.00280-13

Dordet-Frisoni E, Marenda MS, Sagné E, et al (2013b) ICEA of Mycoplasma agalactiae: a new family of self-transmissible integrative elements that confers conjugative properties to the recipient strain. Mol Microbiol 89:1226-1239. doi: 10.1111/ mmi.12341

Dordet-Frisoni E, Sagné E, Baranowski E, et al (2014) Chromosomal transfers in mycoplasmas: when minimal genomes go mobile. mBio 5:e01958. doi: 10.1128/mBio.01958-14

Dubey GP, Ben-Yehuda S (2011) Intercellular nanotubes mediate bacterial communication. Cell 144:590-600. doi: 10.1016/j.cell.2011.01.015

Felsenstein J (1992) Estimating effective population size from samples of sequences: a bootstrap Monte Carlo integration method. Genet Res 60:209-220

Frost LS, Leplae R, Summers AO, Toussaint A (2005) Mobile genetic elements: the agents of open source evolution. Nat Rev Microbiol 3:722-732. doi: 10.1038/nrmicro1235

Gasparich GE, Whitcomb RF, Dodge D, et al (2004) The genus Spiroplasma and its non-helical descendants: phylogenetic classification, correlation with phenotype and roots of the Mycoplasma mycoides clade. Int J Syst Evol Microbiol 54:893-918. doi: 10.1099/ijs.0.02688-0

Gibson DG, Glass JI, Lartigue C, et al (2010) Creation of a bacterial cell controlled by a chemically synthesized genome. Science 329:52-56. doi: 10.1126/science.1190719

Gray TA, Clark RR, Boucher N, et al (2016) Intercellular communication and conjugation are mediated by ESX secretion systems in mycobacteria. Science 354:347-350. doi: 10.1126/ science.aag0828

Gray TA, Krywy JA, Harold J, et al (2013) Distributive conjugal transfer in mycobacteria generates progeny with meiotic-like genome-wide 
mosaicism, allowing mapping of a mating identity locus. PLoS Biol 11:e1001602. doi: 10.1371/ journal.pbio.1001602

Guérillot R, Siguier P, Gourbeyre E, et al (2014) The diversity of prokaryotic DDE transposases of the mutator superfamily, insertion specificity, and association with conjugation machineries. Genome Biol Evol 6:260-272. doi: 10.1093/gbe/ evu010

Guglielmini J, Quintais L, Garcillán-Barcia MP, et al (2011) The repertoire of ICE in prokaryotes underscores the unity, diversity, and ubiquity of conjugation. PLoS Genet 7:e1002222. doi: 10.1371/journal.pgen.1002222

Halary S, Leigh JW, Cheaib B, et al (2010) Network analyses structure genetic diversity in independent genetic worlds. Proc Natl Acad Sci U S A 107:127-132. doi: 10.1073/pnas.0908978107

Hochhut B, Marrero J, Waldor MK (2000) Mobilization of plasmids and chromosomal DNA mediated by the SXT element, a constin found in Vibrio cholerae 0139. J Bacteriol 182:2043-2047

Jaenike J, Unckless R, Cockburn SN, et al (2010) Adaptation via symbiosis: recent spread of a Drosophila defensive symbiont. Science 329:212-215. doi: 10.1126/science.1188235

Johansson, Petterson (2002) Phylogeny and Evolution. In: Molecular biology and pathogenicity of Mycoplasmas, Kluwer Academic / Plenum Publishers. S. Razin and R. Herrmann, New York (USA), pp P1-29

Johnson CM, Grossman AD (2015) Integrative and Conjugative Elements (ICEs): What they do and how they work. Annu Rev Genet. doi: 10.1146/ annurev-genet-112414-055018

Kloesges T, Popa O, Martin W, Dagan T (2011) Networks of gene sharing among 329 proteobacterial genomes reveal differences in lateral gene transfer frequency at different phylogenetic depths. Mol Biol Evol 28:1057-1074. doi: $10.1093 / \mathrm{molbev} / \mathrm{msq} 297$

Komano T, Yoshida T, Narahara K, Furuya N (2000) The transfer region of Incl1 plasmid R64: similarities between R64 tra and Legionella icm/ dot genes. Mol Microbiol 35:1348-1359

Koski LB, Morton RA, Golding GB (2001) Codon bias and base composition are poor indicators of horizontally transferred genes. Mol Biol Evol 18:404-412

Ku C, Lo W-S, Chen L-L, Kuo C-H (2013) Complete genomes of two dipteran-associated spiroplasmas provided insights into the origin, dynamics, and impacts of viral invasion in spiroplasma. Genome Biol Evol 5:1151-1164. doi: 10.1093/gbe/evt084
Kumar S, Stecher G, Tamura K (2016) MEGA7: Molecular evolutionary genetics analysis version 7.0 for bigger datasets. Mol Biol Evol 33:1870-1874. doi: $10.1093 / \mathrm{molbev} / \mathrm{msw} 054$

Kuo C-H (2015) Scrambled and not-so-tiny genomes of fungal endosymbionts. Proc Natl Acad Sci U S A 112:7622-7623. doi: 10.1073/ pnas. 1508360112

Kuo C-H, Ochman H (2009a) Deletional bias across the three domains of life. Genome Biol Evol 1:145-152. doi: 10.1093/gbe/evp016

Kuo C-H, Ochman H (2010) The extinction dynamics of bacterial pseudogenes. PLoS Genet 6:. doi: 10.1371/journal.pgen.1001050

Kuo C-H, Ochman H (2009b) The fate of new bacterial genes. FEMS Microbiol Rev 33:38-43. doi: 10.1111/j.1574-6976.2008.00140.x

Lederberg J, Tatum EL (1946) Gene recombination in Escherichia coli. Nature 158:558

Lesic B, Zouine M, Ducos-Galand M, et al (2012) A natural system of chromosome transfer in Yersinia pseudotuberculosis. PLoS Genet 8:e1002529. doi: 10.1371/journal.pgen.1002529

Liu W, Feng Z, Fang L, et al (2011) Complete genome sequence of Mycoplasma hyopneumoniae strain 168. J Bacteriol 193:1016-1017. doi: 10.1128/JB.01305-10

Llosa M, Gomis-Rüth FX, Coll M, de la Cruz Fd F (2002) Bacterial conjugation: a two-step mechanism for DNA transport. Mol Microbiol $45: 1-8$

Lo W-S, Chen L-L, Chung W-C, et al (2013a) Comparative genome analysis of Spiroplasma melliferum IPMB4A, a honeybee-associated bacterium. BMC Genomics 14:22. doi: 10.1186/1471-2164-14-22

Lo W-S, Gasparich GE, Kuo C-H (2015) Found and lost: The fates of horizontally acquired genes in arthropod-symbiotic Spiroplasma. Genome Biol Evol 7:2458-2472. doi: 10.1093/gbe/evv160

Lo W-S, Huang Y-Y, Kuo C-H (2016) Winding paths to simplicity: genome evolution in facultative insect symbionts. FEMS Microbiol Rev. doi: 10.1093/femsre/fuw028

Lo W-S, Ku C, Chen L-L, et al (2013b) Comparison of metabolic capacities and inference of gene content evolution in mosquito-associated Spiroplasma diminutum and $S$. taiwanense. Genome Biol Evol 5:1512-1523. doi: 10.1093/gbe/ evt108

Lo W-S, Kuo C-H (2017) Horizontal acquisition and transcriptional integration of novel genes in mosquito-associated Spiroplasma. Genome Biol Evol 9:3246-3259. doi: 10.1093/gbe/evx244 
Manso-Silván L, Tardy F, Baranowski E, et al (2013) Draft genome sequences of Mycoplasma alkalescens, Mycoplasma arginini, and Mycoplasma bovigenitalium, three species with equivocal pathogenic status for cattle. Genome Announc 1:. doi: 10.1128/genomeA.00348-13

Mardassi BBA, Aissani N, Moalla I, et al (2012) Evidence for the predominance of a single tet(M) gene sequence type in tetracycline-resistant Ureaplasma parvum and Mycoplasma hominis isolates from Tunisian patients. J Med Microbiol 61:1254-1261. doi: 10.1099/jmm.0.044016-0

Marenda M, Barbe V, Gourgues G, et al (2006) A new integrative conjugative element occurs in Mycoplasma agalactiae as chromosomal and free circular forms. J Bacteriol 188:4137-4141. doi: 10.1128/JB.00114-06

Marenda SM (2014) Genomic mosaics. In: Mollicutes, Molecular Biology and Pathogenesis, Caister Academic Press. Browning G., Citti C., Norfolk, UK, pp 15-54

Marques LM, Guimarães AMS, Martins HB, et al (2015) Genome sequence of Ureaplasma diversum strain ATCC 49782. Genome Announc 3:. doi: 10.1128/genomeA.00314-15

Marques LM, Rezende IS, Barbosa MS, et al (2016) Ureaplasma diversum genome provides new insights about the interaction of the surface molecules of this bacterium with the host. PloS One 11:e0161926. doi: 10.1371/journal.pone. 0161926

McCutcheon JP, Moran NA (2011) Extreme genome reduction in symbiotic bacteria. Nat Rev Microbiol 10:13-26. doi: 10.1038/nrmicro2670

Minion FC, Lefkowitz EJ, Madsen ML, et al (2004) The genome sequence of Mycoplasma hyopneumoniae strain 232, the agent of swine mycoplasmosis. J Bacteriol 186:7123-7133. doi: 10.1128/JB.186.21.7123-7133.2004

Moran NA, Bennett GM (2014) The tiniest tiny genomes. Annu Rev Microbiol 68:195-215. doi: 10.1146/annurev-micro-091213-112901

Naito M, Morton JB, Pawlowska TE (2015) Minimal genomes of mycoplasma-related endobacteria are plastic and contain host-derived genes for sustained life within Glomeromycota. Proc Natl Acad Sci U S A 112:7791-7796. doi: 10.1073/ pnas. 1501676112

Naito M, Pawlowska TE (2016) The role of mobile genetic elements in evolutionary longevity of heritable endobacteria. Mob Genet Elem 6:e1136375. doi: 10.1080/2159256X.2015.1136375

Naumann M, Schüssler A, Bonfante P (2010) The obligate endobacteria of arbuscular mycorrhizal fungi are ancient heritable components related to the Mollicutes. ISME J 4:862-871. doi: 10.1038/ ismej.2010.21

Noormohammadi AH, Markham PF, Duffy MF, et al (1998) Multigene families encoding the major hemagglutinins in phylogenetically distinct mycoplasmas. Infect Immun 66:3470-3475

Nouvel LX, Sirand-Pugnet P, Marenda MS, et al (2010) Comparative genomic and proteomic analyses of two Mycoplasma agalactiae strains: clues to the macro- and micro-events that are shaping mycoplasma diversity. BMC Genomics 11:86. doi: 10.1186/1471-2164-11-86

Nunan LM, Lightner DV, Oduori MA, Gasparich GE (2005) Spiroplasma penaei sp. nov., associated with mortalities in Penaeus vannamei, Pacific white shrimp. Int J Syst Evol Microbiol 55:2317-2322. doi: 10.1099/ijs.0.63555-0

Ochman H, Lawrence JG, Groisman EA (2000) Lateral gene transfer and the nature of bacterial innovation. Nature 405:299-304. doi: 10.1038/35012500

Ozbek E, Miller SA, Meulia T, Hogenhout SA (2003) Infection and replication sites of Spiroplasma kunkelii (Class: Mollicutes) in midgut and Malpighian tubules of the leafhopper Dalbulus maidis. J Invertebr Pathol 82:167-175

Paralanov V, Lu J, Duffy LB, et al (2012) Comparative genome analysis of 19 Ureaplasma urealyticum and Ureaplasma parvum strains. BMC Microbiol 12:88. doi: 10.1186/1471-2180-12-88

Paredes JC, Herren JK, Schüpfer F, et al (2015) Genome sequence of the Drosophila melanogaster male-killing Spiroplasma strain MSRO endosymbiont. mBio 6:. doi: 10.1128/ mBio.02437-14

Pereyre S, Sirand-Pugnet P, Beven L, et al (2009) Life on arginine for Mycoplasma hominis: clues from its minimal genome and comparison with other human urogenital mycoplasmas. PLoS Genet 5:e1000677. doi: 10.1371/journal.pgen. 1000677

Pinto PM, Carvalho MO de, Alves-Junior L, et al (2007) Molecular analysis of an integrative conjugative element, ICEH, present in the chromosome of different strains of Mycoplasma hyopneumoniae. Genet Mol Biol 30:256-263. doi: 10.1590/S1415-47572007000200014

Rabel C, Grahn AM, Lurz R, Lanka E (2003) The VirB4 family of proposed traffic nucleoside triphosphatases: Common motifs in plasmid RP4 TrbE are essential for conjugation and phage adsorption. J Bacteriol 185:1045-1058. doi: 10.1128/JB.185.3.1045-1058.2003

Razin S, Hayflick L (2010) Highlights of mycoplasma research--an historical perspective. Biol J Int 
Assoc Biol Stand 38:183-190. doi: 10.1016/ j.biologicals.2009.11.008

Roberts AP, Mullany P (2011) Tn916-like genetic elements: a diverse group of modular mobile elements conferring antibiotic resistance. FEMS Microbiol Rev 35:856-871. doi: 10.1111/j. 1574-6976.2011.00283.x

Rocha EPC, Cornet E, Michel B (2005) Comparative and evolutionary analysis of the bacterial homologous recombination systems. PLoS Genet 1:e15. doi: 10.1371/journal.pgen. 0010015

Saillard C, Carle P, Duret-Nurbel S, et al (2008) The abundant extrachromosomal DNA content of the Spiroplasma citri GII3-3X genome. BMC Genomics 9:195. doi: 10.1186/1471-2164-9-195

Saitou N, Nei M (1987) The neighbor-joining method: a new method for reconstructing phylogenetic trees. Mol Biol Evol 4:406-425

Shu H-W, Liu T-T, Chan H-I, et al (2012) Complexity of the Mycoplasma fermentans M64 genome and metabolic essentiality and diversity among mycoplasmas. PloS One 7:e32940. doi: 10.1371/ journal.pone.0032940

Sirand-Pugnet P, Citti C, Barré A, Blanchard A (2007a) Evolution of mollicutes: down a bumpy road with twists and turns. Res Microbiol 158:754-766. doi: 10.1016/j.resmic.2007.09.007

Sirand-Pugnet $\mathrm{P}$, Lartigue $\mathrm{C}$, Marenda $\mathrm{M}$, et al (2007b) Being pathogenic, plastic, and sexual while living with a nearly minimal bacterial genome. PLoS Genet 3:e75. doi: 10.1371/ journal.pgen.0030075

Szpirer C, Top E, Couturier M, Mergeay M (1999) Retrotransfer or gene capture: a feature of conjugative plasmids, with ecological and evolutionary significance. Microbiol Read Engl 145:3321-3329

Tamura K, Nei M, Kumar S (2004) Prospects for inferring very large phylogenies by using the neighbor-joining method. Proc Natl Acad Sci U S A 101:11030-11035. doi: 10.1073/pnas.0404206101

Tardy F, Mick V, Dordet-Frisoni E, et al (2015) Integrative conjugative elements are widespread in field isolates of Mycoplasma species pathogenic for ruminants. Appl Environ Microbiol 81:1634-1643. doi: 10.1128/AEM.03723-14

Teachman AM, French CT, Yu H, et al (2002) Gene transfer in Mycoplasma pulmonis. J Bacteriol 184:947-951

Thiaucourt F, Manso-Silvan L, Salah W, et al (2011) Mycoplasma mycoides, from "mycoides Small Colony" to "capri". A microevolutionary perspective. BMC Genomics 12:114. doi: 10.1186/1471-2164-12-114
Tinsley MC, Majerus MEN (2006) A new male-killing parasitism: Spiroplasma bacteria infect the ladybird beetle Anisosticta novemdecimpunctata (Coleoptera: Coccinellidae). Parasitology 132:757-765. doi: 10.1017/S0031182005009789

Torres-Cortés G, Ghignone S, Bonfante P, Schüßler A (2015) Mosaic genome of endobacteria in arbuscular mycorrhizal fungi: Transkingdom gene transfer in an ancient mycoplasma-fungus association. Proc Natl Acad Sci U S A 112:7785-7790. doi: 10.1073/pnas.1501540112

Tully JG (1982) Interaction of spiroplasmas with plant, arthropod, and animal hosts. Rev Infect Dis 4 Suppl:S193-199

Tully JG, Bove JM, Laigret F, et al (1993) Revised taxonomy of the class Mollicutes: proposed elevation of a monophyletic cluster of arthropodassociated mollicutes to ordinal rank (Entomoplasmatalesord. nov.), with Provision for familial rank to separate species with nonhelical morphology (Entomoplasmataceaefam. nov.) from helical species (Spiroplasmataceae), and emended descriptions of the order Mycoplasmatales, family Mycoplasmataceae

Tully JG, Whitcomb RF, Williamson DL, Clark HF (1976) Suckling mouse cataract agent is a helical wall-free prokaryote (spiroplasma) pathogenic for vertebrates. Nature 259:117-120

Vasconcelos ATR, Ferreira HB, Bizarro CV, et al (2005) Swine and poultry pathogens: the complete genome sequences of two strains of Mycoplasma hyopneumoniae and a strain of Mycoplasma synoviae. J Bacteriol 187:5568-5577. doi: 10.1128/ JB.187.16.5568-5577.2005

Voelker LL, Dybvig K (1996) Gene transfer in Mycoplasma arthritidis: transformation, conjugal transfer of Tn916, and evidence for a restriction system recognizing $A G C T$. J Bacteriol 178:6078-6081

Wang B (2001) Limitations of compositional approach to identifying horizontally transferred genes. J Mol Evol 53:244-250. doi: 10.1007/ s002390010214

Wang W, Gu W, Gasparich GE, et al (2011) Spiroplasma eriocheiris sp. nov., associated with mortality in the Chinese mitten crab, Eriocheir sinensis. Int J Syst Evol Microbiol 61:703-708. doi: 10.1099/ijs.0.020529-0

Wise KS, Calcutt MJ, Foecking MF, et al (2011) Complete genome sequence of Mycoplasma bovis type strain PG45 (ATCC 25523). Infect Immun 79:982-983. doi: 10.1128/IAI.00726-10

Wozniak RAF, Waldor MK (2010) Integrative and conjugative elements: mosaic mobile genetic elements enabling dynamic lateral gene flow. Nat 
Rev Microbiol 8:552-563. doi: 10.1038/ nrmicro2382

Xiao L, Paralanov V, Glass JI, et al (2011) Extensive horizontal gene transfer in ureaplasmas from humans questions the utility of serotyping for diagnostic purposes. J Clin Microbiol 49:2818-2826. doi: 10.1128/JCM.00637-11

Xiao L, Ptacek T, Osborne JD, et al (2015) Comparative genome analysis of Mycoplasma pneumoniae. BMC Genomics 16:610. doi: 10.1186/s12864-015-1801-0
Xie J, Tiner B, Vilchez I, Mateos M (2011) Effect of the Drosophila endosymbiont Spiroplasma on parasitoid wasp development and on the reproductive fitness of wasp-attacked fly survivors. Evol Ecol 53:1065-1079. doi: 10.1007/ s10682-010-9453-7

Xie J, Vilchez I, Mateos M (2010) Spiroplasma bacteria enhance survival of Drosophila hydei attacked by the parasitic wasp Leptopilina heterotoma. PloS One 5:e12149. doi: 10.1371/ journal.pone.0012149. 